\title{
Modeling and Simulation of Three-Phase Voltage Source Inverter with Dynamic Phasors
}

\author{
Yi Li ${ }^{1, a}$, Xinhua Yang ${ }^{2, b}$, Lizhen Wu ${ }^{3, c}$, Shike Zhang ${ }^{4, d}$ \\ 1,2,3,4College of Electrical and Information Engineering, Lanzhou University of Technology, Lanzhou, \\ China \\ aliy_laura2017@163.com, byangxh5852@163.com, cwulzh@lut.cn, d18394177095@163.com
}

\begin{abstract}
Keywords: three-phase voltage source inverter, dynamic phasor method, dynamic phasor model, EMT model
\end{abstract}

Abstract. The rapid switching of the high-frequency three-phase voltage source inverter bring the system with the rapid dynamic process, which beyond the application of the traditional phasor model. Furthermore, a large number of power electronic devices make the electromagnetic transient (EMT) model of microgrid involving multiple inverters very complex. In this paper, the dynamic model of three-phase voltage source inverter (VSI) interfacing with micro-source is established using dynamic phasor method under the condition of RL loads. The three-phase VSI model is simplified by keeping important system state variables corresponding to the time-varying Fourier series, which include switching function considering both the DC component and fundamental frequency component, and the DC source considering only the DC component, the AC output considering only the fundamental frequency component. Finally, the dynamic phasor (DP) model of the three-phase VSI and its EMT model was built in Matlab/Simulink. By comparing the DP model and the detailed time domain EMT model of three phase VSI, the simulation results show that the DP model can reflect the main dynamic characteristics, improve the simulation speed and reduce the running time.

\section{Introduction}

Microgrid is emerging to be an effective approach for the integration of various types of distributed energy. Three-phase voltage source inverters as an important component of energy conversion and energy management in microgrid, however, they have small inertia and high-frequency nonlinear characteristics, so its modeling research is of great significance.

The quasi steady state model in power system transient analysis can not accurately describe the fast dynamic process of the three-phase VSI in microgrid; the differential equation model in time domain can accurately reflect the electromagnetic transient process of the system, which is often used to test whether other models are correct, but its nonlinear and time-varying characteristics is not suitable for the analysis of system containing a large number of power electronic devices. Dynamic phasors broke the quasi-steady-state assumption of the traditional phasor, could be used to establish dynamic phasor model whose accuracy between time-domain model and quasi-steady model, and could analyze the fast dynamic process. Dynamic phasors was based on the classical state space averaging, it was introduced in [1] for the first time, and the model of the series resonant converter was established by preserving those effective Fourier series coefficients of the system variables. The dynamic phasors then was applied to the modeling and analysis of power system ${ }^{[2-4]}$ TCSC $^{[5]}$, DC/DC converters ${ }^{[6]}$ and single phase inverters ${ }^{[7]}$.

In this paper, dynamic phasors is employed to model the high-frequency three-phase VSI based on its switching function model. This model greatly reduces the computational complexity and ensures the simulation accuracy by preserving the significant system state variables corresponding to the time-varying Fourier series. As a result, the high frequency switching process is largely simplified. This method not only greatly reduces the computational cost, but also ensures the accuracy of the simulation. 


\section{The Concept of Dynamic Phasors}

When the variable does not meet the sinusoidal steady state or quasi-steady state assumption, that is, a non-sinusoidal signal, its instantaneous value can also be decomposed into a series of different frequencies, different amplitudes, different phase signal superposition by Fourier transform. A possible complex time-domain waveform $x(\tau)$ can be represented on the interval $\tau \in(t-T, t)$ by the Fourier series

$$
x(\tau)=\sum_{k=-\infty}^{\infty} X_{k}(t) e^{j k \omega_{s} \tau},
$$

Where $\omega_{s}=2 \pi / T$ and $X_{k}(t)$ are the complex Fourier coefficients. Notice that these coefficients are functions of time and are given by

$$
X_{k}(t)=\frac{1}{T} \int_{t-T}^{t} x(\tau) e^{-j k \omega_{s} \tau} d \tau=\langle x\rangle_{k}(t) .
$$

The notation used to indicate dependence of the dynamic phasor states on time, ' $(\mathrm{t})$ ', is omitted for convenience. That is to say, $\langle x\rangle_{k}$ is the kth time-dependent Fourier complex coefficient, also defined as kth dynamic phasors.

There are two key and useful properties of dynamic phasors:

(1)Computation of average of product

The index-k average of the product of signals $x(t)$ and $q(t)$ can be computed by the following formula:

$$
\langle x q\rangle_{k}=\sum_{i=-\infty}^{\infty}\langle x\rangle_{k-i}\langle q\rangle_{i} .
$$

(2)Differentiation with respect to time

The derivative with respect to time of the kth coefficient of the Fourier series can be calculated by the following relationship:

$$
\frac{d\langle x\rangle_{k}}{d t}=-j k \omega_{s}\langle x\rangle_{k}+\left\langle\frac{d x}{d t}\right\rangle_{k} .
$$

Compared to the traditional signal differential nature, the second term of the formula (4) preserves a part of the dynamic characteristics of the signal.

Dynamic phasor method based on frequency decomposition theory approximates the original waveform with a very small number of Fourier coefficients. Certainly, the more harmonics be contained in the model, the more accurate the model will be, but the complexity of the model will be significantly increased. In practical applications, in order to simplify the derivation of the model and reduce the computational burden, the relational large Fourier coefficients of the model can be determined according to the needs of the study. The dynamic phasor model performs an average operation on the time-domain differential equation, which is a large-signal model with slow change over time. Thus, the dynamic phasor model can be simulated with larger simulation step size.

\section{Dynamic Phasor Model of Three-phase Voltage Source Inverter}

The main circuit of three-phase voltage source inverter is shown in Fig. 1. Since the dynamic of the DC link is not the main problem to be discussed in this paper, it is replaced by a DC voltage source. The switch function is defined as when $S_{j}=1$, the switches of the upper arm are on; When $S_{j}=0$, the switches of the lower arm are on, where $j=a, b, c$. Although there are many types of loads that can exist in a three-phase voltage source inverter, the general three-phase symmetrical RL load is considered in this paper. 


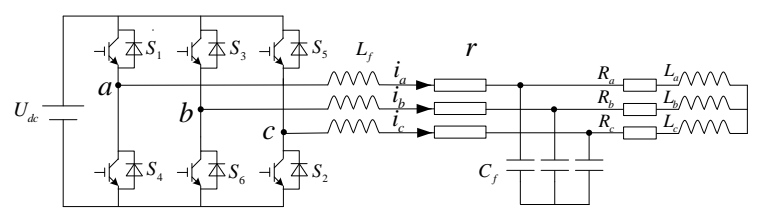

Fig. 1. Main circuit diagram of three-phase voltage source inverter

The Kirchhoff voltage and current laws of the circuit depicted in Fig. 1 allow to write the following equations

$$
\begin{aligned}
& L_{f} \frac{d i_{a}}{d t}=-r i_{a}-u_{c a}-\frac{u_{d c}}{3} \sum_{j=a, b, c} S_{j}+u_{d c} * S_{a}, \\
& L_{f} \frac{d i_{b}}{d t}=-r i_{b}-u_{c b}-\frac{u_{d c}}{3} \sum_{j=a, b, c} S_{j}+u_{d c} * S_{b}, \\
& L_{f} \frac{d i_{c}}{d t}=-r i_{c}-u_{c c}-\frac{u_{d c}}{3} \sum_{j=a, b, c} S_{j}+u_{d c} * S_{c}, \\
& C_{f} \frac{d u_{c a}}{d t}=i_{a}-\frac{u_{c a}}{R_{a}+j \omega_{s} L_{a}}, \\
& C_{f} \frac{d u_{c b}}{d t}=i_{b}-\frac{u_{c b}}{R_{b}+j \omega_{s} L_{b}}, \\
& C_{f} \frac{d u_{c c}}{d t}=i_{c}-\frac{u_{c c}}{R_{c}+j \omega_{s} L_{c}},
\end{aligned}
$$

Where $L_{f}, C_{f}$ are the filter inductance and the filter capacitance. Further, $R_{k}, L_{k}, i_{k}$ and $u_{c k}$ are the resistive load, the inductive load, the currents flowing through filter inductance and the line voltages, where $k=a, b, c$.

Assuming that the dc variables can be described by the dc components; and the ac variables can be described accurately by their fundamental frequency components. We take into account the dc and fundamental frequency components for the switching functions. Based on these simplifications, the dynamic phasor model of three-phase voltage source inverter is

$$
\begin{aligned}
& \frac{d\left\langle i_{a}\right\rangle_{1}}{d t}=-j \omega_{s}\left\langle i_{a}\right\rangle_{1}+\frac{1}{L_{f}}\left(-r\left\langle i_{a}\right\rangle_{1}-\left\langle u_{c a}\right\rangle_{1}-U_{d c}\left(\sum_{j=a, b, c} S_{j}-S_{a}\right\rangle_{1}\right), \\
& \frac{d\left\langle i_{b}\right\rangle_{1}}{d t}=-j \omega_{s}\left\langle i_{b}\right\rangle_{1}+\frac{1}{L_{f}}\left(-r\left\langle i_{b}\right\rangle_{1}-\left\langle u_{c b}\right\rangle_{1}-U_{d c}\left(\sum_{j=a, b, c} S_{j}-S_{b}\right\rangle_{1}\right), \\
& \frac{d\left\langle i_{c}\right\rangle_{1}}{d t}=-j \omega_{s}\left\langle i_{c}\right\rangle_{1}+\frac{1}{L_{f}}\left(-r\left\langle i_{c}\right\rangle_{1}-\left\langle u_{c c}\right\rangle_{1}-U_{d c}\left(\sum_{j=a, b, c} S_{j}-S_{c}\right\rangle_{1}\right), \\
& \frac{d\left\langle u_{c a}\right\rangle_{1}}{d t}=-j \omega_{s}\left\langle u_{c a}\right\rangle_{1}+\frac{1}{C_{f}}\left(\left\langle i_{a}\right\rangle_{1}-\frac{\left\langle u_{c a}\right\rangle_{1}}{R_{a}+j \omega_{s} L_{a}}\right), \\
& \frac{d\left\langle u_{c b}\right\rangle_{1}}{d t}=-j \omega_{s}\left\langle u_{c b}\right\rangle_{1}+\frac{1}{C_{f}}\left(\left\langle i_{b}\right\rangle_{1}-\frac{\left\langle u_{c b}\right\rangle_{1}}{R_{b}+j \omega_{s} L_{b}}\right), \\
& \frac{d\left\langle u_{c c}\right\rangle_{1}}{d t}=-j \omega_{s}\left\langle u_{c c}\right\rangle_{1}+\frac{1}{C_{f}}\left(\left\langle i_{c}\right\rangle_{1}-\frac{\left\langle u_{c c}\right\rangle_{1}}{R_{c}+j \omega_{s} L_{c}}\right),
\end{aligned}
$$

The fundamental ac components of $S_{a}, S_{b}$ and $S_{c}$ in the whole cycle are 


$$
\begin{aligned}
& d_{a}=0.5+\frac{m}{2} \cos \left(\omega_{s} t-\delta_{1}\right), \\
& d_{b}=0.5+\frac{m}{2} \cos \left(\omega_{s} t-\delta_{1}-\frac{2 \pi}{3}\right), \\
& d_{c}=0.5+\frac{m}{2} \cos \left(\omega_{s} t-\delta_{1}-\frac{4 \pi}{3}\right) .
\end{aligned}
$$

According to the formula (2) of dynamic phasors, the corresponding 0th and 1th dynamic phasors of (7) are

$$
\begin{array}{ll}
\left\langle d_{a}\right\rangle_{0}=\frac{1}{2}, \quad\left\langle d_{a}\right\rangle_{1}=\frac{m}{4} e^{-j \delta_{1}}, \quad & \left\langle d_{b}\right\rangle_{0}=\frac{1}{2}, \\
\left\langle d_{b}\right\rangle_{1}=\frac{m}{4} e^{-j\left(\delta_{1}+\frac{2 \pi}{3}\right)}, \quad\left\langle d_{c}\right\rangle_{0}=\frac{1}{2}, & \left\langle d_{c}\right\rangle_{1}=\frac{m}{4} e^{-j\left(\delta_{1}+\frac{4 \pi}{3}\right)} .
\end{array}
$$

By using (8) in (6) we obtain the dynamic phasor model of the three-phase voltage source inverter:

$$
\begin{aligned}
& \frac{d\left\langle i_{a}\right\rangle_{1}}{d t}=-j \omega_{s}\left\langle i_{a}\right\rangle_{1}+\frac{1}{L_{f}}\left(-r\left\langle i_{a}\right\rangle_{1}-\left\langle u_{c a}\right\rangle_{1}+\frac{m}{4} U_{d c} e^{-j \delta_{1}}\right), \\
& \frac{d\left\langle i_{b}\right\rangle_{1}}{d t}=-j \omega_{s}\left\langle i_{b}\right\rangle_{1}+\frac{1}{L_{f}}\left(-r\left\langle i_{b}\right\rangle_{1}-\left\langle u_{c b}\right\rangle_{1}+\frac{m}{4} U_{d c} e^{-j\left(\delta_{1}+\frac{2 \pi}{3}\right)}\right), \\
& \frac{d\left\langle i_{c}\right\rangle_{1}}{d t}=-j \omega_{s}\left\langle i_{c}\right\rangle_{1}+\frac{1}{L_{f}}\left(-r\left\langle i_{c}\right\rangle_{1}-\left\langle u_{c c}\right\rangle_{1}+\frac{m}{4} U_{d c} e^{-j\left(\delta_{1}+\frac{4 \pi}{3}\right)}\right), \\
& \frac{d\left\langle u_{c a}\right\rangle_{1}}{d t}=-j \omega_{s}\left\langle u_{c a}\right\rangle_{1}+\frac{1}{C_{f}}\left(\left\langle i_{a}\right\rangle_{1}-\frac{\left\langle u_{c a}\right\rangle_{1}}{R_{a}+j \omega_{s} L_{a}}\right), \\
& \frac{d\left\langle u_{c b}\right\rangle_{1}}{d t}=-j \omega_{s}\left\langle u_{c b}\right\rangle_{1}+\frac{1}{C_{f}}\left(\left\langle i_{b}\right\rangle_{1}-\frac{\left\langle u_{c b}\right\rangle_{1}}{R_{b}+j \omega_{s} L_{b}}\right), \\
& \frac{d\left\langle u_{c c}\right\rangle_{1}}{d t}=-j \omega_{s}\left\langle u_{c c}\right\rangle_{1}+\frac{1}{C_{f}}\left(\left\langle i_{c}\right\rangle_{1}-\frac{\left\langle u_{c c}\right\rangle_{1}}{R_{c}+j \omega_{s} L_{c}}\right) .
\end{aligned}
$$

By representing the phasors in (9) as the form of real part and imaginary part, we get the complete dynamic phasor model of three-phase voltage source inverter:

$$
\begin{aligned}
& \frac{d\left\langle i_{a}\right\rangle_{1}^{R}}{d t}=\omega_{s}\left\langle i_{a}\right\rangle_{1}^{I}+\frac{1}{L_{f}}\left[-r\left\langle i_{a}\right\rangle_{1}^{R}-\left\langle u_{c a}\right\rangle_{1}^{R}+\frac{m}{4} U_{d c} \cdot \sin \left(\delta_{1}+\frac{\pi}{2}\right)\right], \\
& \frac{d\left\langle i_{a}\right\rangle_{1}^{I}}{d t}=-\omega_{s}\left\langle i_{a}\right\rangle_{1}^{R}+\frac{1}{L_{f}}\left[-r\left\langle i_{a}\right\rangle_{1}^{I}-\left\langle u_{c a}\right\rangle_{1}^{I}-\frac{m}{4} U_{d c} \cdot \sin \delta_{1}\right], \\
& \frac{d\left\langle i_{b}\right\rangle_{1}^{R}}{d t}=\omega_{s}\left\langle i_{b}\right\rangle_{1}^{I}+\frac{1}{L_{f}}\left[-r\left\langle i_{b}\right\rangle_{1}^{R}-\left\langle u_{c b}\right\rangle_{1}^{R}+\frac{m}{4} U_{d c} \cdot \sin \left(\delta_{1}+\frac{7 \pi}{6}\right)\right], \\
& \frac{d\left\langle i_{b}\right\rangle_{1}^{I}}{d t}=-\omega_{s}\left\langle i_{b}\right\rangle_{1}^{R}+\frac{1}{L_{f}}\left[-r\left\langle i_{b}\right\rangle_{1}^{I}-\left\langle u_{c b}\right\rangle_{1}^{I}-\frac{m}{4} U_{d c} \cdot \sin \left(\delta_{1}+\frac{2 \pi}{3}\right)\right], \\
& \frac{d\left\langle i_{c}\right\rangle_{1}^{R}}{d t}=\omega_{s}\left\langle i_{c}\right\rangle_{1}^{I}+\frac{1}{L_{f}}\left[-r\left\langle i_{c}\right\rangle_{1}^{R}-\left\langle u_{c c}\right\rangle_{1}^{R}+\frac{m}{4} U_{d c} \cdot \sin \left(\delta_{1}+\frac{11 \pi}{6}\right)\right],
\end{aligned}
$$




$$
\begin{aligned}
& \frac{d\left\langle i_{c}\right\rangle_{1}^{I}}{d t}=-\omega_{s}\left\langle i_{c}\right\rangle_{1}^{R}+\frac{1}{L_{f}}\left[-r\left\langle i_{c}\right\rangle_{1}^{I}-\left\langle u_{c c}\right\rangle_{1}^{I}-\frac{m}{4} U_{d c} \cdot \sin \left(\delta_{1}+\frac{4 \pi}{3}\right)\right], \\
& \frac{d\left\langle u_{c a}\right\rangle_{1}^{R}}{d t}=\omega_{s}\left\langle u_{c a}\right\rangle_{1}^{I}+\frac{1}{C_{f}}\left(\left\langle i_{a}\right\rangle_{1}^{R}-\frac{R_{a}\left\langle u_{c a}\right\rangle_{1}^{R}+\omega_{s} L_{a}\left\langle u_{c a}\right\rangle_{1}^{I}}{R_{a}^{2}+\left(\omega_{s} L_{a}\right)^{2}}\right), \\
& \frac{d\left\langle u_{c a}\right\rangle_{1}^{I}}{d t}=-\omega_{s}\left\langle u_{c a}\right\rangle_{1}^{R}+\frac{1}{C_{f}}\left(\left\langle i_{a}\right\rangle_{1}^{I}-\frac{\left.R_{a}\left\langle u_{c a}\right\rangle_{1}^{I}-\omega_{s} L_{a}\left\langle u_{c a}\right\rangle_{1}^{R}\right),}{R_{a}^{2}+\left(\omega_{s} L_{a}\right)^{2}}\right) \\
& \frac{d\left\langle u_{c b}\right\rangle_{1}^{R}}{d t}=\omega_{s}\left\langle u_{c b}\right\rangle_{1}^{I}+\frac{1}{C_{f}}\left(\left\langle i_{b}\right\rangle_{1}^{R}-\frac{R_{b}\left\langle u_{c b}\right\rangle_{1}^{R}+\omega_{s} L_{b}\left\langle u_{c b}\right\rangle_{1}^{I}}{\left.R_{b}^{2}+\left(\omega_{s} L_{b}\right)^{2}\right),}\right. \\
& \frac{d\left\langle u_{c b}\right\rangle_{1}^{I}}{d t}=-\omega_{s}\left\langle u_{c b}\right\rangle_{1}^{R}+\frac{1}{C_{f}}\left(\left\langle i_{b}\right\rangle_{1}^{I}-\frac{R_{b}\left\langle u_{c b}\right\rangle_{1}^{I}-\omega_{s} L_{b}\left\langle u_{c b}\right\rangle_{1}^{R}}{R_{b}^{2}+\left(\omega_{s} L_{b}\right)^{2}}\right), \\
& \frac{d\left\langle u_{c c}\right\rangle_{1}^{R}}{d t}=\omega_{s}\left\langle u_{c c}\right\rangle_{1}^{I}+\frac{1}{C_{f}}\left(\left\langle i_{c}\right\rangle_{1}^{R}-\frac{R_{c}\left\langle u_{c c}\right\rangle_{1}^{R}+\omega_{s} L_{c}\left\langle u_{c c}\right\rangle_{1}^{I}}{R_{c}^{2}+\left(\omega_{s} L_{c}\right)^{2}}\right), \\
& \frac{d\left\langle u_{c c}\right\rangle_{1}^{I}}{d t}=-\omega_{s}\left\langle u_{c c}\right\rangle_{1}^{R}+\frac{1}{C_{f}}\left(\left\langle i_{c}\right\rangle_{1}^{I}-\frac{R_{c}\left\langle u_{c c}\right\rangle_{1}^{I}-\omega_{s} L_{c}\left\langle u_{c c}\right\rangle_{1}^{R}}{R_{c}^{2}+\left(\omega_{s} L_{c}\right)^{2}}\right) .
\end{aligned}
$$

\section{Simulation Results}

The three-phase VSI is controlled by amplitude controller[8], and the reference voltage is $311 \mathrm{~V}$. The dynamic phasor model of the system shown in Fig. 1 is constructed by employing signal processing module in MATLAB/simulink, and the corresponding electromagnetic transient model is established by utilizing SimPowerSystems module in MATLAB/simulink. The simulations have been carried out by considering these realistic electrical parameters values shown in Table 1 . When $t=0.052 \mathrm{~s}$, the disturbance of $-60 \Omega$ is introduced at loads. Simulation results of the current flowing through the filter inductor and the output voltage of the system are shown in Fig. 2.

Table 1. Electrical parameters of three-phase voltage source inverter

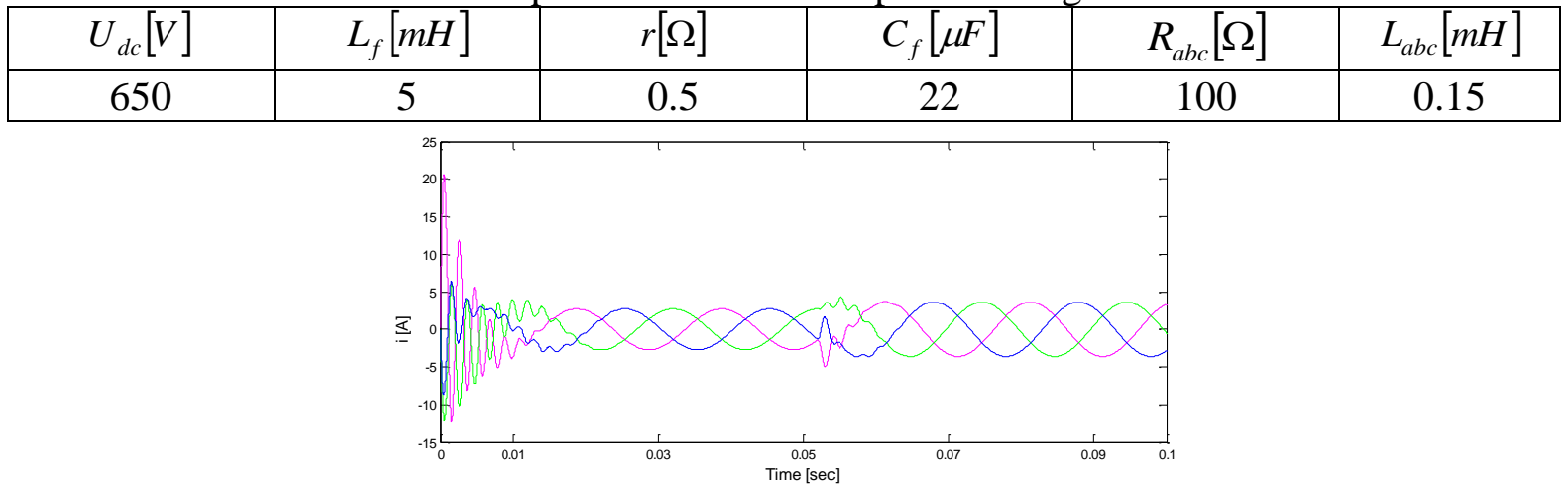

Fig. 2. Inductor current waveform in the DP model

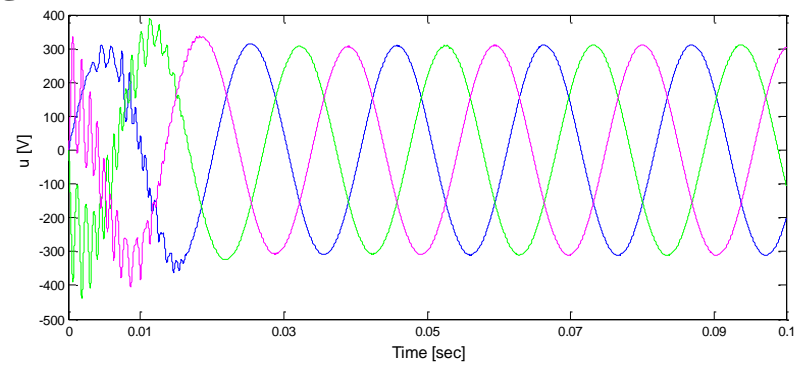

Fig. 3. Output voltage waveform in the DP model 


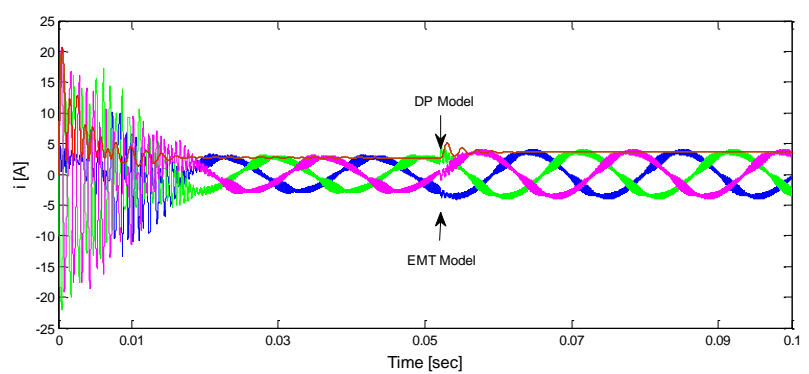

Fig. 4. Comparison of inductor current waveform

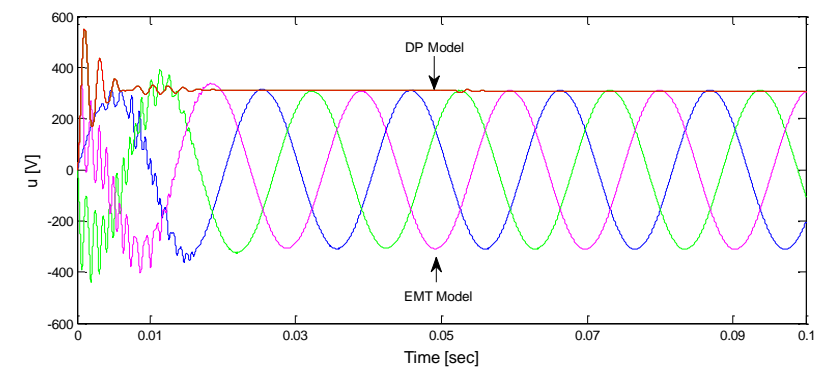

Fig. 5. Comparison of output voltage waveform

Fig. 2 and Fig. 3 severally show the waveform of the filtered inductor current and the output voltage in the EMT model. Fig. 4 and Fig. 5 present the waveform of the inductor current and the output voltage in the EMT model, respectively. It can be seen from above simulation results that the DP model which retains the dc and the fundamental frequency components is agree with the EMT model on the amplitude and the trend. Thus, we could retain the required harmonics according to different actual needs. Furthermore, in Fig. 4 and Fig. 5, the waveform of dynamic phasor model are the amplitude of the inductor current and output voltage, which are the envelope of the simulation results in the EMT model. Compared to the actual curve, it changes slowly, we can employ larger integration step in the system simulation to improve speed. The simulation step size of the DP model is $2 \mu \mathrm{s}$, and the simulation run time is 3.8s. The simulation step size of the EMT model is $0.1 \mu$ s and the simulation run time is $144.1 \mathrm{~s}$.

\section{Conclusions}

In this paper, the dynamic phasor model of high frequency three-phase voltage source inverter with RL load is deduced and verified by simulation. Simulation results suggest that the established dynamic phasor model not only reflects the steady-state characteristics of the three-phase voltage source inverter, but also well reflects the main dynamic process; besides, the established dynamic phasor model is suitable for the RL load; in addition, the established dynamic phasor model could employ a larger simulation step, which can speed up the simulation speed and significantly reduce the simulation time. Therefore, the dynamic phasor method is more appropriate to model the system with a large number of power electronic devices.

\section{Acknowledgements}

This work was financially supported by the National Natural Science Foundation of China (No.51467009), the Project of Lanzhou Science and Technology Plan (No. 2016-3-67).

\section{References}

[1] Sanders S R, Noworolski J M, Liu X Z, et al. IEEE Transactions on Power Electronics, 1991, 6(2): 251-259.

[2] DeMarco C L. Bringing phasor dynamics into the power system load flow[M]. University of Wisconsin, Engineering Experiment Station, 1993. 
[3] Venkatasubramanian V. International Journal of Electrical Power \& Energy Systems, 1994, 16(6): 365-376.

[4] Venkatasubramanian V, Schattler H, Zaborszky J. IEEE Transactions on Automatic Control, 1995, 40(11): 1975-1982.

[5] Mattavelli P, Stankovic A M, Verghese G C. IEEE Transactions on Power Systems, 1999, 14(1): 200-208.

[6] Peng Zhang, Biying Zhou, Yuyuan Wang, et al. Modern Electric Power, 2011, 28(1): 41-44.(In Chinese)

[7] Gu H R, An S C, Zhao W, et al. Modeling and Simulation of Single Phase Inverter with Dynamic Phasors[C]//Advanced Materials Research. Trans Tech Publications, 2012, 354: 1381-1385.

[8] Bala S, Venkataramanan G. On the choice of voltage regulators for droop-controlled voltage source converters in microgrids to ensure stability[C]//Energy Conversion Congress and Exposition (ECCE), 2010 IEEE. IEEE, 2010: 3448-3455. 